Bio - grafía. Escritos sobre la Biología y su Enseñanza. ISSN 2027-1034

Edición Extraordinaria. p.p. 1443-1456

Memorias del IX Encuentro Nacional de Experiencias en Enseñanza de la Biología y la Educación Ambiental. IV Congreso Nacional de Investigación en Enseñanza de la Biología.

\title{
VISIÓN DE LA CIENCIA: ¿COINCIDE EL ALUMNADO CON EL PROFESORADO DE CIENCIAS?
}

\section{VIEW OF SCIENCE: DO STUDENTS AGREE WITH SCIENCE TEACHERS?}

\author{
Andrea García Torres \\ Marta Talavera \\ Olga Mayoral Berlanga ${ }^{1}$
}

\section{RESUMEN}

El aprendizaje de conceptos relacionados con las ciencias y la Educación Ambiental está marcado por la actitud que el alumnado presenta hacia las ciencias. En el presente trabajo se realiza un estudio sobre la actitud que presenta el alumnado y profesorado de primer curso de la Educación Secundaria Obligatoria (ESO) hacia las ciencias, concretamente, hacia la asignatura de Biología y Geología a través de la realización de cuestionarios actitudinales. La disposición que presenta el alumnado hacia las tareas y actividades escolares, en este caso relacionadas con las ciencias, encuentra parte de su origen en la disposición que transmite y ven reflejadas en el profesorado, pudiendo éste llegar a modificar la actitud y motivación que presentará el alumnado hacia la asignatura. Se puede decir que la actitud mostrada por el alumnado objeto de estudio es en general buena, no presentándose diferencias significativas en cuanto al género. En el caso del propio profesorado, la actitud frente a la enseñanza de ciencias en clase es muy buena, se puede decir que la parte extrínseca de la motivación del alumnado está, en este caso, cubierta por la motivación que llegan a observar en su profesor.

PALABRAS CLAVE: actitud, motivación, ciencias, biología y geología

\begin{abstract}
The present work is a study on the attitudes shown by the students and teachers of Compulsory Secondary School (ESO in Spanish) towards science, specifically, towards Biology and Geology subject through the accomplishment of attitudinal questionnaires. Part of the disposition students present to school tasks and activities, in this case related to science, finds its origin in the attitude shown by their teachers, which could even modify the motivation to the students towards the subject. The attitude shown by the studied alumni is generally good, with no significant differences in gender. Teacher's attitude towards the

\footnotetext{
${ }^{1}$ Universidad de Valencia. (España).
} 
teaching of science is really good. In this sense, it could be said that the extrinsic part of the students' motivation is covered by the motivation they receive from their teacher.

KEY WORDS: attitude, motivation, science, biology and geology

\section{INTRODUCCIÓN}

Desde hace bastante tiempo se viene indicando que el aprendizaje de conceptos científicos abarca aspectos epistemológicos, cognitivos y lingüísticos (Astolfi, 1987). Por ello, la enseñanza y aprendizaje de estos conceptos, es un proceso complejo, que requiere un modelo que integre todos los aspectos y que considere que los estudiantes, en su proceso de aprendizaje, se involucrarán y comprometerán con este proceso en la medida en que establezcan relaciones con otros semejantes, de forma que los conocimientos les ayuden a sentir su pertenencia a un colectivo (Gallego \& Pérez, 1997).

De este modo, a la hora de introducir cualquier concepto nuevo en la Enseñanza de las Ciencias, se deben conocer las concepciones previas de las que partimos, como afirmaban Ausubel, Novak \& Hanesian (1983), ya que es la única manera en que es posible emplear las ideas previamente aprendidas en el procesamiento de ideas nuevas, relacionándolas con las primeras. Es importante abordar así mismo su nivel de aproximación al conocimiento científico, para saber cuáles de las nociones que puedan considerarse como correctas pueden ser ampliadas durante el proceso de enseñanza-aprendizaje o por el contrario, si existen ideas alternativas al conocimiento científico, que deben ser profundamente modificadas (Guerrero, 2006).

A todo esto debemos añadir la predisposición, tanto de los docentes a enseñar ciencias como del alumnado para aprenderlas. En la actualidad, dentro de la enseñanza de las ciencias se considera importante hacerlas llegar a todos los alumnos como algo útil, relacionado con la vida real y enseñar una ciencia escolar relevante para el ciudadano (Acevedo, 2004) y esta idea debe ser transmitida por el propio docente, especialmente si estamos hablando de temas de Educación Ambiental que implican a todo el entorno.

Así, la intención del presente trabajo es conocer la actitud del alumnado hacia la enseñanza de las ciencias naturales y la biología, y la del docente y detectar si existen diferencias de género entre las diferentes actitudes.

\section{METODOLOGÍA}

Para conocer la actitud del alumnado y profesorado frente a las ciencias, se diseñó un cuestionario que tiene en cuenta la percepción que tiene el alumnado frente a la asignatura de Biología y Geología en diferentes aspectos de su vida como son: las clases, en casa, y en la sociedad. Este cuestionario fue validado y pilotado previamente a su utilización en este estudio. 
Por otra parte, el profesorado respondió a un cuestionario basado en otro redactado por García-Ruiz \& Orozco (2008) en el que se tienen en cuenta las inquietudes, formación y sentimientos que produce la enseñanza de las ciencias en dicho profesorado.

La muestra del presente estudio es el alumnado y profesorado de la asignatura de Biología y Geología de 1ำ de Educación Secundaria Obligatoria (ESO) en una región de España. El alumnado encuestado (143 estudiantes) pertenecía a primer curso de la ESO. Dado que los resultados a los cuestionarios se recogieron de forma anónima, cada cuestionario respondido se clasifica según la franja horaria en la que se realizó y se rechaza un cuestionario respondido fuera del horario escolar, por no poder adjudicarlo a ninguna sección. Las clases presentan gran variedad de alumnado según origen socioeconómico y situación familiar, siendo la proporción de alumnado inmigrante minoritario. Algo más de la mitad del alumnado encuestado son chicos $(n=85: 59,4 \%)$ y un poco menos de la mitad son chicas ( $n=58 ; 40,5 \%)$, por lo que se puede afirmar que la muestra está equilibrada en cuando a género. Las edades se sitúan entre 11 y 13 años. Respecto al profesorado, se encuestó a los docentes de la asignatura de Biología y Geología de este mismo curso, de todas las secciones.

Los instrumentos para llevar a cabo el presente estudio son dos cuestionarios para medir la actitud hacia las ciencias, que se pasaron en formato digital con la aplicación google drive, Formularios de Google.

Tras los correspondientes permisos del director de Secundaria, y garantizando el anonimato del alumnado, se pidió a las clases objeto de estudio que cumplimentara un cuestionario original, en el que se valoraban aspectos relacionados con la actitud que presenta hacia las ciencias.

Para poder medir la motivación intrínseca hacia la biología y geología, se redactaron una serie de frases que afirman que generalmente se divierten aprendiendo temas científicos, que les gusta leer sobre ciencia, que son felices trabajando en temas científicos, que les gusta adquirir nuevos conceptos científicos y que están interesados en aprender sobre ciencia. Se debía seleccionar el grado de acuerdo mediante 4 opciones de respuesta, desde 'nada de acuerdo' a 'completamente de acuerdo'. El cuestionario se dividía en tres principales componentes: la actitud que presentan en clase, la que mantienen en casa, y la que presentan en la vida (Anexo I).

Por otra parte, también se pasó un cuestionario actitudinal al profesor de Biología y Geología de 1ํ de ESO (Anexo II), en el que se le pedía señalar una serie de ítems según su grado de acuerdo con adjetivos respecto a su sentir hacia la enseñanza de las ciencias y el trabajo de la comunidad científica, entre otros. 


\section{RESULTADOS Y DISCUSIÓN}

\section{Alumnado}

En primer lugar, se analizan las respuestas del alumnado al cuestionario de la actitud que presentan hacia la asignatura de Biología y Geología.

\section{Resultados a los ítems cuantitativos por género}

El primer bloque del cuestionario actitudinal pedía al alumnado mostrar su grado de acuerdo con respecto a una serie de frases que afirman que les gusta la asignatura de Biología y Geología, que disfrutan realizando los trabajos y tareas de la asignatura, o que les gusta hablar de temas científicos con familia y amigos, entre otras. Los valores van del 1, nada de acuerdo, hasta el máximo de 4 puntos, totalmente de acuerdo, en un total de 15 ítems de esta primera parte.

Entre los mejor valorados, los chicos coinciden con las chicas en la valoración de tres ítems con los que están más de acuerdo ( $\mathrm{M}, \mathrm{F}$ y O$)^{2}$. Además, los chicos valoran positivamente otros ítems que refuerzan su creencia en la utilidad de las ciencias para la sociedad $(\mathrm{L}$ y $\mathrm{N})$.

Para los peor valorados, los chicos coinciden con las chicas en varios ítems. Esto refleja que, en general, no disfrutan haciendo tareas relacionadas con la asignatura en casa, no mejora o incrementa la información que consideran interesante -o quizá ni la encuentran- y que en clase, en general, no acuden para solucionar dudas (Figura 1).

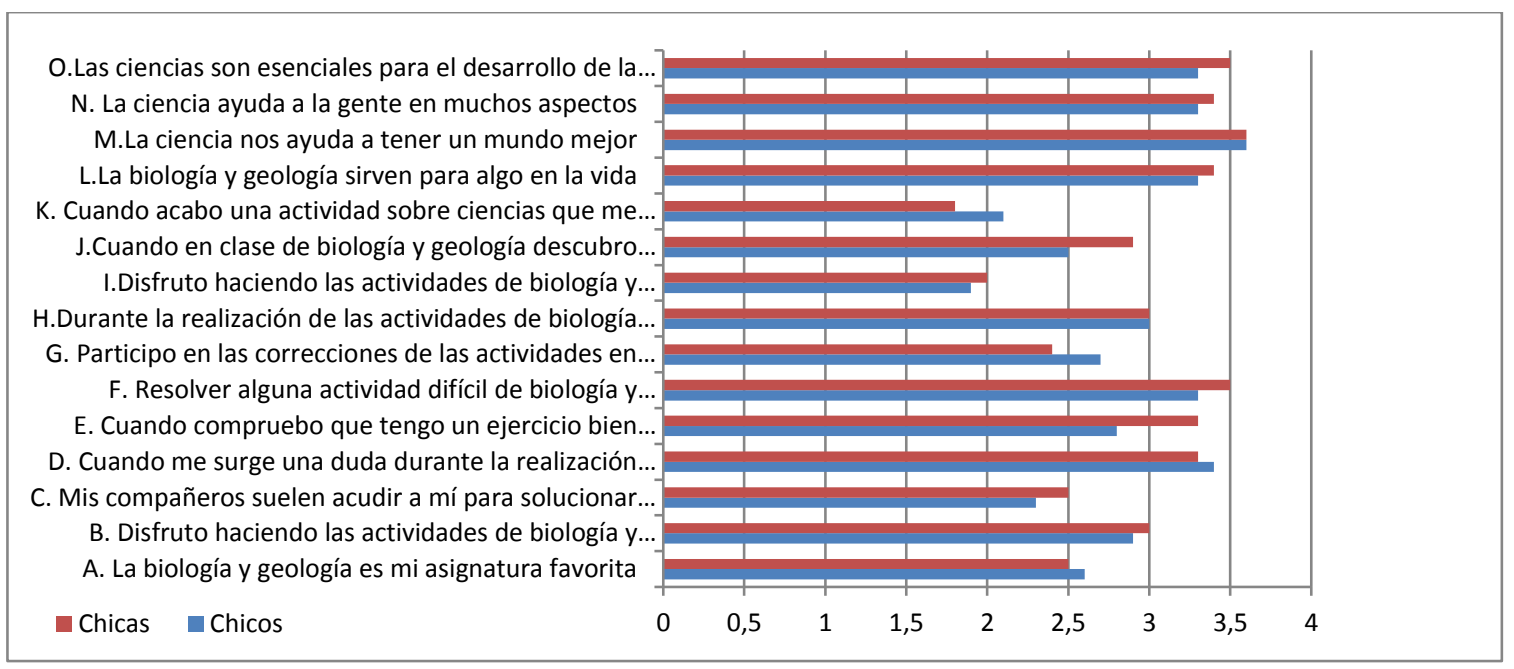

Figura 1. Valoración de diferentes enunciados sobre ciencia. Por géneros.

\footnotetext{
${ }^{2}$ Las siglas corresponden a los enunciados de la tabla 1
} 


\section{Resultados a los ítems cualitativos por género}

Las cuestiones de carácter cualitativo siguen haciendo referencia a los aspectos anteriormente nombrados (en el colegio, en casa y en la sociedad), para seguir conociendo la actitud del alumnado de $1^{\circ}$ de ESO en estos diferentes ámbitos.

\section{Respuestas a las preguntas abiertas:}

\section{- ¿ ¿Ves documentales de televisión sobre ciencia? ¿Cuáles?}

Poco más de la mitad de las chicas encuestadas afirma no ver documentales de televisión relacionados con la ciencia. Entre aquellas que sí los ven, las respuestas más comunes son: El Hormiguero $(n=4)$, documentales de plantas y animales $(n=4)$ y Discovery Max $(n=3)$. Por otra parte, aparecen programas considerados por tratar temas científicos como: Cuarto Milenio $(n=1)$ o la serie Big Bang Theory $(\mathrm{n}=1)$.

Poco más de la mitad de los chicos encuestados afirma no ver programas relacionados con la ciencia. Los programas que más aparecen son: El Hormiguero $(n=8)$, documentales de La $2(n=6)$, National Geographic $(n=3)$, Discovery Max $(n=3)$ y documentales de animales $(n=3)$. También entre los chicos aparece la serie Big Bang Theory $(n=1)$.

En conjunto, se puede observar en ambas gráficas que aproximadamente la mitad del alumnado que respondió a esta pregunta afirma no ver programas relacionados con temas científicos, 71 de un total de 127.

\section{- ¿ ¿Hablas con tus padres sobre temas científicos? Pon ejemplos}

El segundo ítem pregunta al alumnado si hablan con sus padres sobre temas científicos, y, en caso afirmativo, que indique cuáles son esos temas. De esta forma, y de igual modo que en el ítem anterior, se obtiene información de la idea que el alumnado tiene sobre lo que son temas científicos.

Se observa que más de la mitad de las chicas afirma no hablar de temas científicos en casa. De aquellas que afirmar sí hacerlo, la mayoría coincide en hablar sobre los deberes o temas que actualmente están estudiando en clase $(n=9)$, si bien una menor proporción de chicas habla en casa de temas científicos debido a las profesiones de sus padres -un padre biólogo, padre y madre médicos- $(n=2)$. Hay alumnas que hablan en casa de temas no relacionados con la asignatura directamente, y no debido a la profesión de sus padres $(n=10)$.

El caso de los chicos no es tan equitativo como el de las chicas. Como se observa en la gráfica superior, casi tres cuartos de los chicos encuestados afirman no hablar en casa de temas científicos. De aquellos que sí que lo hacen se encuentran las siguientes respuestas muy variadas: animales $(n=2)$, descubrimientos $(n=1)$, astrofísica $(n=1)$, células $(n=1)$, terremotos $(n=2)$. Además de temas relacionados directamente con el temario actual de la asignatura $(n=7)$.

\section{- ¿Hablas con tus amigos sobre temas científicos? Pon ejemplos}


El tercer ítem preguntaba si se habla con el resto de alumnado de clase sobre temas científicos. Si bien la mayoría responde que no $(n=108)$, una pequeña proporción sí afirma hablar de temas científicos, manifestando en su mayoría, que se trata de temas relacionados con la asignatura o alguna tarea que estén desarrollando en el momento $(n=16)$.

- ¿ ¿Tienes juegos o juguetes que tengan que ver con las ciencias? ¿Cuáles?

En el cuarto ítem, sobre juegos y juguetes, se opta por no tener en cuenta cuando no saben cómo se llama el juego, ya que es una contestación muy ambigua. Solo se toma como respuesta válida aquella en la que sí se indica el nombre. Así de las chicas que responden sí, los juguetes o juegos que más aparecen son: cajas de experimentos $(n=7)$, microscopios $(n=5)$, Quimicefa $(n=3)$ y telescopios $(n=2)$ invernadero $(n=2)$. Los juguetes más recurrentes en los chicos son: Quimicefa $(n=8)$, juegos de experimentos $(n=5)$ y telescopios $(n=2)$.

- ¿Conoces alguna revista en la que se hable de ciencia? ¿Cuáles? ¿Lees alguna de ellas?

El quinto ítem pregunta al alumnado si conoce y lee alguna revista científica. En este caso se han tomado tres posibles respuestas: sí conoce y lee alguna revista, no conoce, y por tanto no lee ninguna revista científica, y una tercera opción; conoce, pero no la lee.

Tan solo 10 de las 53 chicas encuestadas responden que sí leen revistas científicas y añaden el nombre, estas son: National Geographic $(n=5)$ y la revista Muy Interesante $(\mathrm{n}=2)$.

Del total de 77 chicos que responden a esta pregunta, solo 9 afirman leer revistas relacionadas con temas científicos, entre estas revistas destacan: Muy Interesante $(\mathrm{n}=3)$ y National Geographic $(\mathrm{n}=2)$.

- ¿Conoces aplicaciones relacionadas con la ciencia? ¿Cuáles? ¿Usas alguna de ellas?

Este ítem cualitativo, pregunta al alumnado si conoce alguna aplicación relacionada con la ciencia y si la utiliza. Se realiza la misma clasificación de resultados que en el punto anterior: conoce y usa; conoce pero no usa; no conoce ni usa. Con diferencia, la gran mayoría de chicas no conoce, ni usa, aplicaciones relacionadas con la ciencia. De las 5 que sí lo hacen se encuentran: Preguntados $(\mathrm{n}=2)$, Mapa Estelar $(\mathrm{n}=1)$ y otras como Stop Disasters $(\mathrm{n}=1)$ usadas en clase. También son pocos los chicos que sí conocen y utilizan aplicaciones científicas, entre las que se encuentran: Stop Disasters $(\mathrm{n}=3)$ o Preguntados $(\mathrm{n}=2)$.

- Pregunta final: ¿Te gustaría seguir estudiando asignaturas relacionadas con las ciencias en el colegio? Si/ no/ me da igual

Por último, se hizo una pregunta al alumnado para conocer su interés por continuar estudiando asignaturas relacionadas con la ciencia en próximos cursos. Se consideró importante incluir la opción 'me da igual', ya que muchos 
estudiantes podían elegir la opción 'sí' por compromiso. De esta forma se les dio la oportunidad de mostrar su indiferencia (Figura 2). Se ve claramente, que es un alumnado minoritario el que no querría continuar estudiando biología y geología en próximos cursos, tanto chicas como chicos. Por otra parte, el número de alumnos y alumnas que sí querría seguir estudiando y les da igual, es muy similar, siendo aquí mayor la diferencia en cuanto al género.

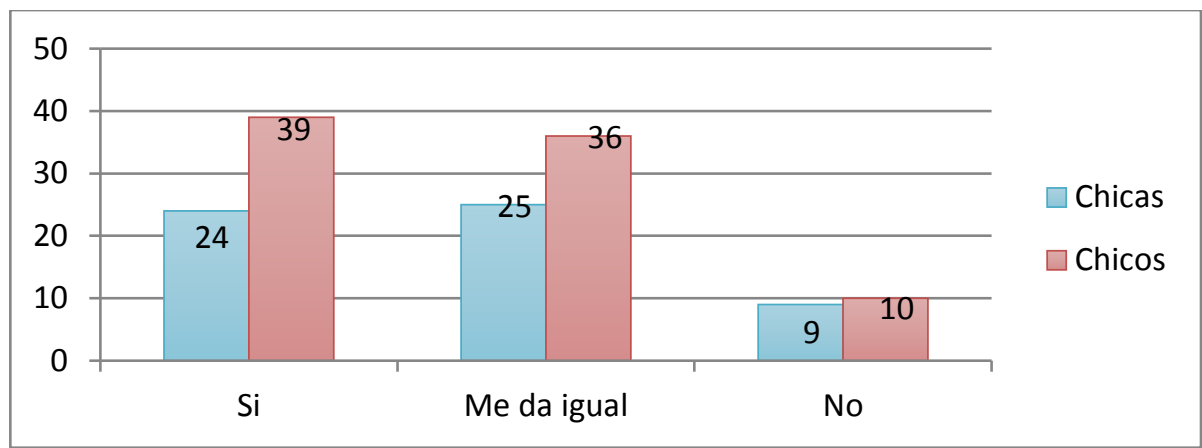

\section{Figura 2. Respuesta a ¿Te gustaría seguir estudiando asignaturas relacionadas con las ciencias en el colegio?}

\section{Profesorado}

Se pasa a continuación a estudiar las respuestas de cada apartado del cuestionario actitudinal del profesorado $(n=5)$. Este cuestionario preguntaba sobre sus sentimientos hacia las ciencias, la enseñanza de las ciencias en clase, la valoración del personal científico, etc. con una escala gradual del 1 (sensación más positiva o atracción) al 4 (sensación negativa o rechazo). (Anexoll).

- ¿Cómo le hacen sentir las ciencias?: En esta primera parte del cuestionario el profesorado escogía todas las emociones positivas, afirmando que las ciencias le hacen sentir atracción, interés, optimismo, etc. entre otras.

- ¿QQué le produce la enseñanza de las ciencias?: En este caso la mayoría de respuestas son cercanas a las sensaciones positivas (tranquilidad, interés, agrado, satisfacción), si bien en la sección a elegir entre confianza y desconfianza selecciona la casilla número 3 , esto es, la mitad justa. De forma general, se puede afirmar que la enseñanza de las ciencias también le provoca sensaciones positivas.

- ¿QQué le parece el trabajo que desarrolla la comunidad científica? En esta sección el profesorado responde que el trabajo que realiza la comunidad científica le parece agradable, divertido, útil y relevante, si bien difícil y mal remunerado.

- ¿QQué le parece plantear experimentos con su alumnado?: Aquí las respuestas ya son más variables, no muy seguro, no muy fácil y entre algo en lo que confía y un desafío. 
- Grado de acuerdo y desacuerdo con una serie de frases: El profesorado afirma que las ciencias son importantes para el desarrollo de la sociedad, si bien puede representar también una amenaza.

\section{CONCLUSIONES}

Una vez analizados los resultados de los test de actitud se pueden obtener las siguientes conclusiones. Por una parte, la actitud del alumnado recién llegado a la Educación Secundaria es buena (2.9/4). Por otra parte, los sentimientos que despiertan las ciencias en el profesor de la asignatura de Biología y Geología son muy positivos, si bien ciertos aspectos pueden presentar un riesgo. Y, por último, no existen diferencias significativas entre chicas y chicos (2,9 puntos) en cuanto a su actitud hacia las ciencias en la clase. Además, la predisposición del profesorado es buena respecto a la enseñanza de las ciencias.

Por tanto, se puede afirmar que la actitud hacia las ciencias, y el aprendizaje de conceptos relacionados con las mismas es positivo y sólo requiere de una metodología adecuada que potencie esta actitud, tal como han señalado otros estudios.

\section{BIBLIOGRAFÍA}

Acevedo, J.A. (2004). Reflexiones sobre las finalidades de la enseñanza de las ciencias: educación científica para la ciudadanía. Revista Eureka sobre Enseñanza y Divulgación de las Ciencias, 1 (1), 3-16.

Astolfi, J.P. (1988). El aprendizaje de conceptos científicos: aspectos epistemológicos, cognitivos y lingüísticos. Enseñanza de las ciencias: revista de investigación y experiencias didácticas, 6 (2), 147-155.

Ausubel, D., Novak, J., \& Hanesian H. (1983). Psicología Educativa. Un punto de vista cognitivo. México: Trillas.

Gallego, R., \& Perez, R. (1997). El concepto de aprendizaje total: Una aproximación. Univ. Pedagógica Nacional: Santafé de Bogotá.

García-Ruíz, M., \& Orozco, L. (2008). Orientando un cambio de actitud hacia las Ciencias Naturales y su enseñanza en Profesores de Educación Primaria. Revista Electrónica de Enseñanza de las Ciencias, 7 (3), 1513-79.

Guerrero Bel, M., Fernández Guerrero, R., \& Fernández Hernández, J.M. (2006). Las ideas previas y su utilización en la enseñanza de las ciencias morfológicas en carreras afines al campo biológico. Tarbiya: Revista de investigación e innovación educativa, 37, 117-124. 
Anexo I. Cuestionario rellenado por el alumnado

Edad:

$\square$ exo: $\square$ Chico Chica

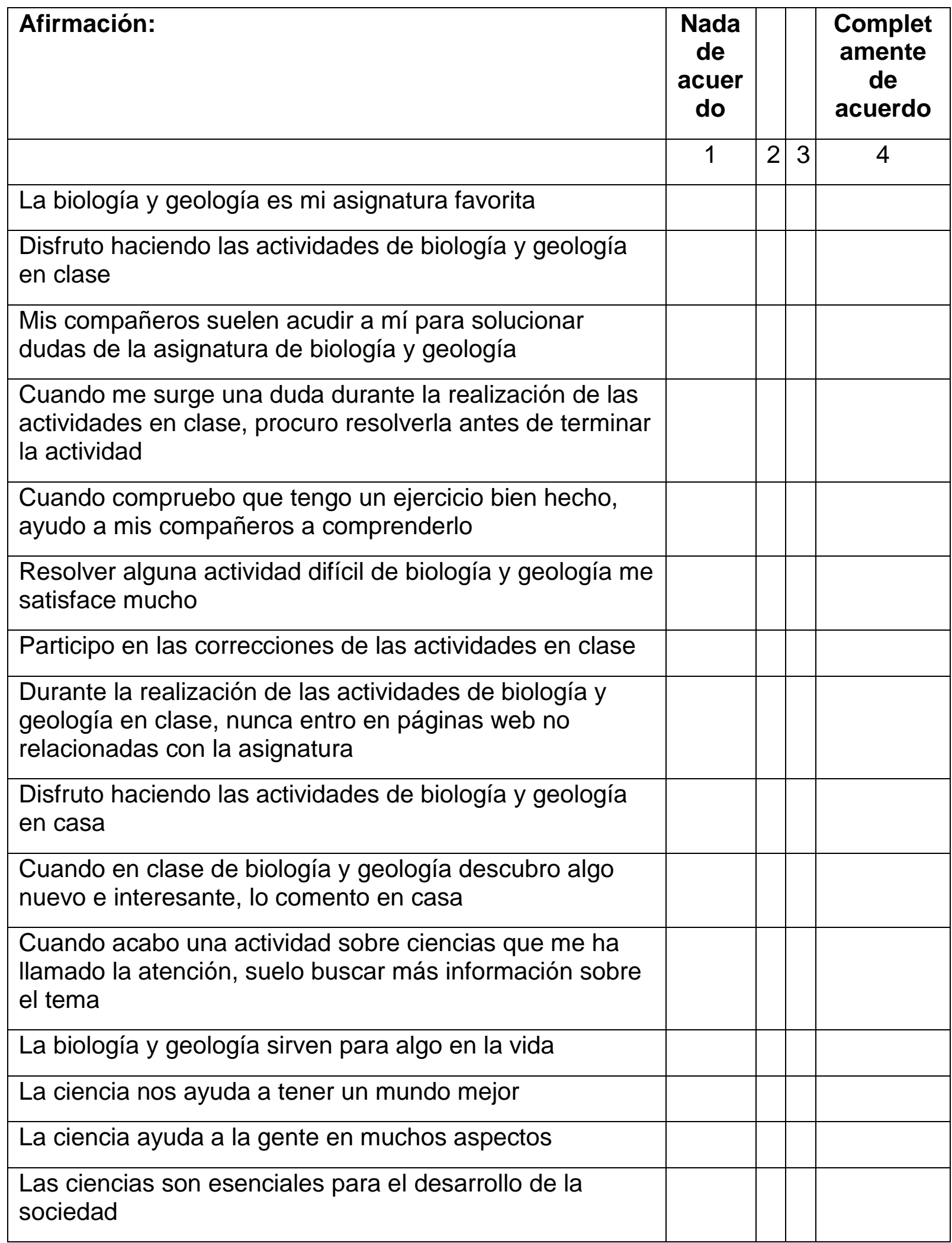


Anexo II. Cuestionario rellenado por el profesorado

Edad:

S $\square \times 0: \quad$ Ma $\square$ ulino

Femenino País de nacimiento:

\begin{tabular}{|l|l|}
\hline \multicolumn{2}{|c|}{ Datos biográficos y académicos } \\
\hline ¿Qué carrera tiene? & \\
\hline $\begin{array}{l}\text { A parte de la titulación para ejercer como profesor/a, ¿tiene algún título } \\
\text { oficial más? }\end{array}$ & \\
\hline $\begin{array}{l}\text { Mencione tres razones por las cuales usted decidió estudiar para } \\
\text { profesor/a }\end{array}$ & \\
\hline ¿Qué asignatura imparte en 1ํ de ESO? & \\
\hline
\end{tabular}

Cuestionario: Seleccione la posición que exprese mejor su sentir con respecto al concepto que hace referencia la siguiente escala:

\begin{tabular}{|l|l|l|l|l|l|l|}
\hline \multicolumn{7}{|l|}{ Las ciencias me hacen sentir: } \\
\hline & $\mathbf{1}$ & $\mathbf{2}$ & $\mathbf{3}$ & $\mathbf{4}$ & $\mathbf{5}$ & \\
\hline Atracción & & & & & & Rechazo \\
\hline Claridad & & & & & & Confusión \\
\hline Entretenimiento & & & & & & Aburrimiento \\
\hline Interés & & & & & & Desinterés \\
\hline Optimismo & & & & & & Pesimismo \\
\hline Agrado & & & & & & Desagrado \\
\hline Motivación & & & & & & Desaliento \\
\hline Apasionamiento & & & & & & Indiferencia \\
\hline La enseñanza de las ciencias me produce: & & & \\
\hline & $\mathbf{1}$ & $\mathbf{2}$ & $\mathbf{3}$ & $\mathbf{4}$ & $\mathbf{5}$ & \\
\hline Tranquilidad & & & & & & Tensión \\
\hline Interés & & & & & & Desinterés \\
\hline Satisfacción & & & & & & Insatisfacción \\
\hline Atracción & & & & & & Rechazo \\
\hline Agrado & & & & & & Desagrado \\
\hline Facilidad & & & & & & Dificultad \\
\hline
\end{tabular}




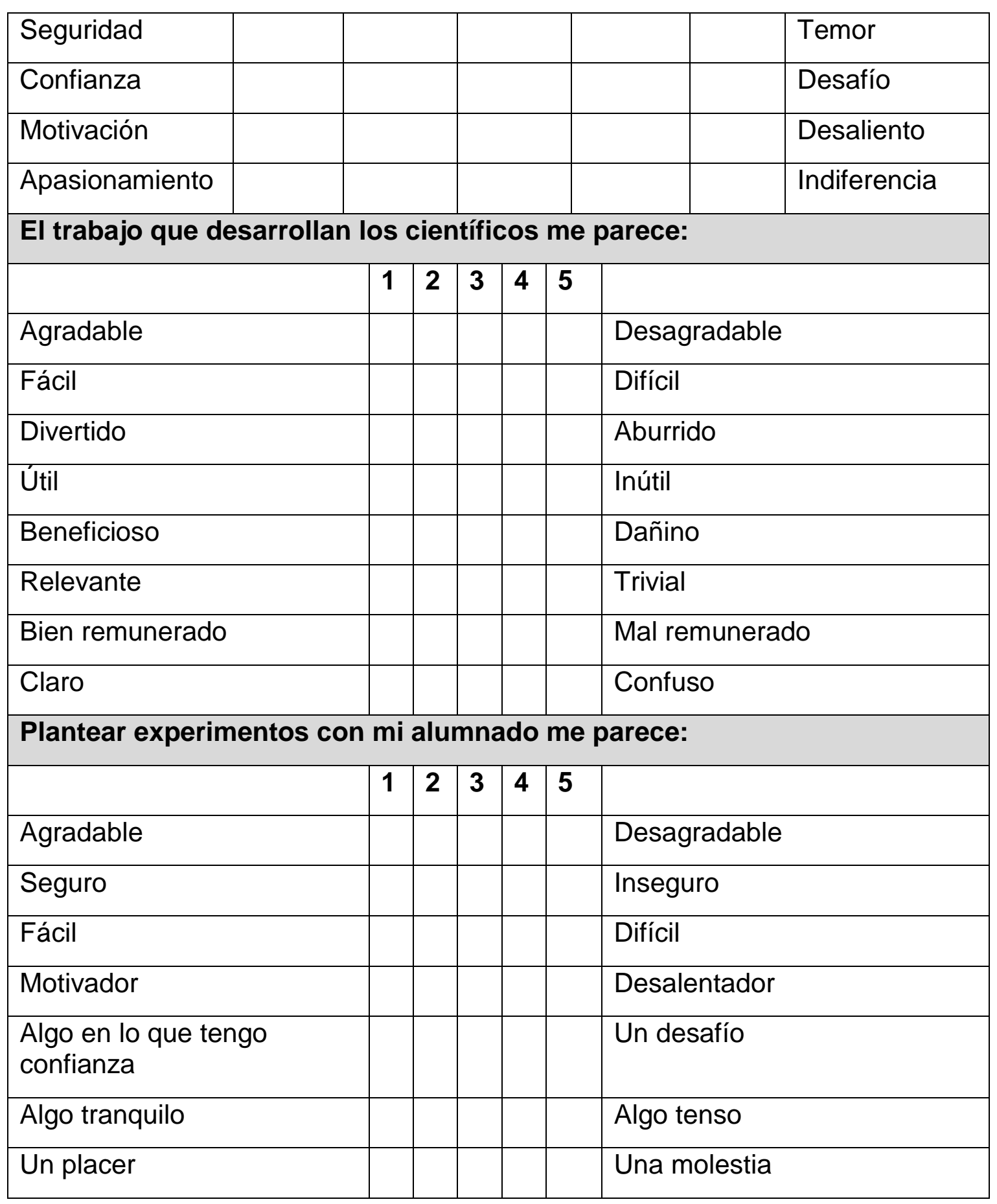

Marque el valor que exprese su grado de acuerdo o desacuerdo con respecto a las afirmaciones que se listan a continuación. ( $1=$ Total acuerdo y $5=$ Total desacuerdo)

\begin{tabular}{|l|l|l|l|l|l|}
\hline Afirmación: & & & & & \\
\hline & 1 & 2 & 3 & 4 & 5 \\
\hline $\begin{array}{l}\text { La ciencia es muy importante para la investigación y desarrollo } \\
\text { de nuestro país. }\end{array}$ & & & & \\
\hline
\end{tabular}




\begin{tabular}{|l|l|l|l|l|}
\hline La ciencia ayuda a que nuestro mundo sea mejor. & & & \\
\hline $\begin{array}{l}\text { La ciencia es el conocimiento cierto, exacto y estático de la } \\
\text { naturaleza. }\end{array}$ & & & & \\
\hline La ciencia puede presentar una amenaza para la sociedad. & & & \\
\hline $\begin{array}{l}\text { Considero que todas las personas deben tener conocimientos } \\
\text { de ciencia en general y de ciencias naturales en particular. }\end{array}$ & & & & \\
\hline $\begin{array}{l}\text { Los objetivos de la investigación científica son comprender la } \\
\text { naturaleza y producir conocimiento. }\end{array}$ & & & \\
\hline La ciencia es un conjunto sistematizado de conocimientos. & & & \\
\hline $\begin{array}{l}\text { Enseñar ciencias es impartir conocimientos que previamente } \\
\text { han sido investigados, experimentados y aceptados. }\end{array}$ & & & \\
\hline $\begin{array}{l}\text { Con el aprendizaje de las ciencias se facilita la comprensión } \\
\text { de otras asignaturas de la educación secundaria. }\end{array}$ & & & \\
\hline $\begin{array}{l}\text { Enseñar ciencias significa que el alumnado comprenda y } \\
\text { verifique sus experiencias cotidianas. }\end{array}$ & & & \\
\hline $\begin{array}{l}\text { La enseñanza de las ciencias no me parece muy útil para } \\
\text { comprender el mundo que nos rodea. }\end{array}$ & & & \\
\hline $\begin{array}{l}\text { La mejor forma de aprender ciencias naturales es mediante la } \\
\text { repetición de los conceptos del parte del profesorado y } \\
\text { alumnado. }\end{array}$ & & & & \\
\hline
\end{tabular}

A partir del siguiente listado, identifique el tipo de actividad de que se trata:

\begin{tabular}{|c|c|c|c|}
\hline Afirmación & $\begin{array}{c}\text { Sí es } \\
\text { una } \\
\text { actividad } \\
\text { científica }\end{array}$ & $\begin{array}{l}\text { Actividad } \\
\text { casi } \\
\text { científica }\end{array}$ & $\begin{array}{c}\text { No es } \\
\text { una } \\
\text { actividad } \\
\text { científica }\end{array}$ \\
\hline $\begin{array}{l}\text { Estudiar el efecto de un fármaco en } \\
\text { personas epilépticas. }\end{array}$ & & & \\
\hline $\begin{array}{l}\text { Elaborar planes y programas de estudio } \\
\text { para el área de Ciencias Naturales. }\end{array}$ & & & \\
\hline $\begin{array}{l}\text { Determinar la relación entre las caricaturas } \\
\text { y las conductas agresivas del alumnado. }\end{array}$ & & & \\
\hline Manejar un ordenador. & & & \\
\hline Estudiar si los OVNIS han visitado la Tierra. & & & \\
\hline $\begin{array}{l}\text { Determinar el índice de criminalidad en la } \\
\text { ciudad de México. }\end{array}$ & & & \\
\hline Tomarle una muestra de sangre a un & & & \\
\hline
\end{tabular}




\begin{tabular}{|l|l|l|l|}
\hline paciente. & & & \\
\hline Estudiar el genoma humano. & & & \\
\hline La clonación de la oveja Dolly. & & & \\
\hline $\begin{array}{l}\text { El diseño y construcción de un aparato } \\
\text { electrodoméstico. }\end{array}$ & & & \\
\hline
\end{tabular}

Posterior al enunciado, se encuentran tres opciones, elija la que considere más cercana a su opinión.

1) El proceso de hacer ciencia se describe mejor como:

i) Todo lo que hacemos para entender el mundo que nos rodea.

ii) El método científico.

iii) Observar y proponer explicaciones sobre las relaciones en el Universo y comprobar la validez de las explicaciones.

2) El gobierno de nuestro país debería dar más dinero a las comunidades científicas para investigar y explorar lo desconocido de la naturaleza y el Universo...

i) Para satisfacer la necesidad humana de conocer lo desconocido, esto es, para cumplir con la curiosidad científica.

ii) Porque comprendiendo mejor nuestro mundo, la comunidad científica podría convertirlo en un lugar mejor para vivir.

iii) No se debe dar más dinero para hacer investigación científica, por las condiciones económicas en las que se encuentra nuestro país.

iv) Para que nuestro país no dependa científicamente de otros.

3) Las autoridades gubernamentales deberían decir a la comunidad científica lo que le corresponde investigar:

i) Sí, para que el trabajo de la comunidad científica ayude a mejorar la sociedad.

ii) La comunidad científica debería tener libertad para decidir qué investigar, porque tienen que mostrar interés en su trabajo para poder ganar creatividad y tener éxito.

iii) Tanto el gobierno como la comunidad científica deben participar por igual para decidir las necesidades que deben estudiarse.

iv) Sí, porque las autoridades gubernamentales conocen mejor los problemas de nuestro país.

4) Para mejorar la calidad de vida de nuestro país, sería mejor gastar dinero en investigación tecnológica en lugar de investigación científica: 
i) Sí, porque mejoraría la producción, el crecimiento económico y el empleo, lo cual es más importante que producir conocimiento científico.

ii) Sí, porque no hay diferencias entre ciencia y tecnología.

iii) No, porque, aunque ambas en determinado momento interaccionan y se complementan, generan diferentes tipos de conocimiento.

iv) Invertir en las dos, porque cada una por su parte ofrece ventajas a la sociedad para mejorar la calidad de vida.

5) El éxito de la ciencia depende de tener un buen equipo científico. Por tanto, nuestro país necesita que el alumnado estudie más ciencias naturales en la escuela:

i) Porque la ciencia afecta a casi todos los aspectos de la sociedad.

ii) Porque la ciencia es importante para que nuestro país tenga un alto nivel de desarrollo.

iii) No, porque son más importantes otras asignaturas (como matemáticas y español) para el éxito futuro de nuestro país.

iv) No, porque no todo el alumnado está interesado en temas científicos. 